\title{
Qualidade de vida e autocuidado em adolescentes com diabetes mellitus tipo 1: uma revisão bibliográfica
}

\author{
Quality of life and self-care in adolescents with type 1 diabetes mellitus: a literature review \\ Calidad de vida y autocuidado en adolescentes con diabetes mellitus tipo 1: \\ revisión de la literatura
}

lasmin Alves Cruz Moy Santana ${ }^{1 *}$, Anselmo Messias Ribeiro da Silva Júnior ${ }^{1}$, Poliana Terra Pires Ribeiro Coelho Caires ${ }^{1}$, Jean Alesi de Aguiar Filho ${ }^{1}$, Rhyan Coelho Santos Souza ${ }^{1}$, Laila Pereira Magalhães ${ }^{1}$, Beatriz de Carvalho Magalhães ${ }^{1}$, Maria Paula dos Anjos Silva ${ }^{1}$, Rebeca Tavares Sampaio Silva ${ }^{1}$, Ícaro Almeida Ladeia ${ }^{1}$.

\section{RESUMO}

Objetivo: Discutir sobre os fatores intervenientes na autonomia dos adolescentes diabéticos tipo 1 e a repercussão desses na qualidade de vida. Métodos: Trata-se de uma Revisão Integrativa de Literatura na qual utilizou-se as bases de dados SciELO e BVS. Os descritores escolhidos foram "diabetes mellitus", "quality of life" e "adolescents", sendo pesquisados com o uso dos operadores booleanos "AND" e "OR". Assim, 23 artigos entre 2010 a 2020 foram escolhidos para análise. Resultados: $O$ adolescente diabético, além de passar pelas alterações psicossociais comuns da fase, deve se adaptar à doença e desenvolver autonomia pelo próprio controle metabólico, o que pode ser um desafio. Imaturidade, medo da dor, baixo poder aquisitivo e superproteção dos pais podem afetar a qualidade de vida e a continuidade do tratamento, aumentando o risco de hospitalizações e complicações graves ou irreversíveis. Considerações finais: O manejo desse grupo de pacientes deve ser feito de forma especial, sendo importante a participação ativa do profissional de saúde, pois o processo do autocuidado deve ser planejado individualmente de modo a contornar os fatores intervenientes na adesão ao tratamento.

Palavras-chave: Diabetes Mellitus tipo 1, Qualidade de vida, Adolescente.

\section{ABSTRACT}

Objective: To discuss the factors involved in the autonomy of type 1 diabetic adolescents and their impact on quality of life. Methods: This is an Integrative Literature Review in which the SciELO and BVS databases were used. The descriptors chosen were "diabetes mellitus", "quality of life" and "adolescents", being researched using the boolean operators "AND" and "OR". Thus, 23 articles between 2010 and 2020 were chosen for analysis. Results: The diabetic adolescent, in addition to going through the common psychosocial changes of the phase, must adapt to the disease and develop autonomy through the metabolic control itself, which can be a challenge. Immaturity, fear of pain, low purchasing power and overprotection of parents can affect quality of life and continuity of treatment, increasing the risk of hospitalizations and serious or irreversible complications. Final considerations: The management of this group of patients must be done in a special way, with the active participation of the health professional being important, since the self-care process must be planned individually in order to circumvent the intervening factors in adherence to treatment.

Keywords: Type 1 Diabetes Mellitus, Quality of life, Adolescent.

\section{RESUMEN}

Objetivo: Discutir los factores involucrados en la autonomía de los adolescentes diabéticos tipo 1 y su impacto en la calidad de vida. Métodos: Se trata de una Revisión de Literatura Integrativa en la que se utilizaron las bases de datos SciELO y BVS. Los descriptores elegidos fueron "diabetes mellitus", "calidad de vida" y "adolescentes", siendo investigados utilizando los operadores booleanos "Y" y "O". Así, se eligieron para el análisis 23 artículos entre 2010 y 2020. Resultados: El adolescente diabético, además de atravesar los cambios psicosociales habituales de la fase, debe adaptarse a la enfermedad y desarrollar autonomía a través del propio control metabólico, lo que puede ser un reto. La inmadurez, el miedo al dolor, el bajo poder adquisitivo y la sobreprotección de los padres pueden afectar la calidad de vida y la continuidad del tratamiento, aumentando el riesgo de hospitalizaciones y complicaciones graves o irreversibles. Consideraciones finales El manejo de este grupo de pacientes debe realizarse de manera especial, siendo importante la participación activa del profesional de la salud, ya que el proceso de autocuidado debe planificarse de manera individual para eludir los factores que intervienen en la adherencia al tratamiento.

Palabras clave: Diabetes Mellitus tipo 1, Calidad de vida, Adolescente.

${ }^{1}$ Faculdades Santo Agostinho (FASA), Vitória da Conquista - BA. *E-mail: iasmin.moy@hotmail.com 


\section{INTRODUÇÃO}

As doenças crônicas são patologias que possuem duração indeterminada, demandando, dessa forma, tratamento e autocuidado contínuos. De acordo com a Organização Mundial de Saúde (OMS), as Doenças Crônicas Não Transmissíveis (DCNT), como desordens cardiovasculares, cerebrovasculares, neoplasias e diabetes mellitus (DM), são as principais responsáveis pelos altos índices de morbimortalidade no mundo (THEME FILHA MM, et al., 2015). No Brasil, cerca de $79,8 \%$ dos óbitos por ano são atribuídos a esse grupo de doenças, além de uma alta taxa de anos de vida perdidos em decorrência da incapacidade que causam (MELO SPSC, et al., 2019).

Por gerar um ciclo vicioso com a pobreza, os países de baixa ou média renda são os mais afetados pelas DCNT. A OMS relata que, em média, $80 \%$ dos óbitos em regiões carentes ocorrem por complicações de curto ou longo prazo dessas patologias, sendo $29 \%$ das mortes em adultos menores de 60 anos. Assim, é evidente o quanto a desigualdade socioeconômica pode acarretar entraves no desenvolvimento de uma nação ao passo que afeta a produtividade e qualidade de vida dos indivíduos (DUNCAN BB, et al., 2012).

À vista disso, percebe-se, então, que as condições socioeconômicas influenciam diretamente na prevalência de DCNT, entretanto é importante evidenciar que essas não são as únicas. Os aspectos genéticos associados à etnia e o sexo bem como o próprio comportamento do indivíduo, no que se diz respeito ao tabagismo, sedentarismo, consumo inadequado de álcool e dietas hipercalóricas, são alguns exemplos dos fatores que estão atrelados a um risco maior de desenvolvimento dessas enfermidades (MENDES TAB, et al., 2011).

Nessa perspectiva, a DM surge como uma síndrome de origem complexa, pois considera aspectos heterogêneos para a sua gênese. De uma maneira geral, é um transtorno caracterizado fundamentalmente por distúrbios metabólicos associado à hiperglicemia e desordens no metabolismo de carboidratos, proteínas e gorduras. Tal fato, ocorre em virtude de uma ação e/ou secreção deficiente de um hormônio, essencial para o bom funcionamento do organismo, chamado insulina (BRASIL, 2013).

Recentemente, as pesquisas no âmbito da Atenção Básica voltaram-se para a importância de inserir novos indicadores em saúde. Assim, além da taxa de mortalidade, a análise da qualidade de vida tornou-se destaque nos novos estudos, pois considera o bem-estar físico, psicológico, socioeconômico e cultural do paciente. Logo, compreender essa mudança de paradigma no processo saúde-doença permite uma discussão tanto da dimensão objetiva médica quanto da dimensão subjetiva do indivíduo, tornando mais eficaz as condutas terapêuticas (MARTINS KAKF, et al., 2018).

Em virtude desses fatos, é de suma importância perceber o DM não apenas na sua concepção biomédica, mas também como uma enfermidade que afeta os indicadores de qualidade de vida, pois impõe limitações nas atividades cotidianas e causa impactos financeiros, gerando estresse, ansiedade e até mesmo transtornos depressivos maiores (RAMOS LBS, et al., 2017). Além disso, a falta do controle glicêmico causa complicações agudas, as quais aumentam o risco de complicações crônicas irreversíveis, influenciando, também, na percepção sobre a qualidade de vida pelos pacientes (GARABELI AA, et al., 2016).

Não é raro, atualmente, se deparar com uma alta prevalência de doenças crônicas, como a DM, em jovens. Pesquisas realizadas no continente europeu evidenciam que em média $3 \%$ a $9 \%$ das crianças possuem DM do tipo 1 (DM1), sendo essa vertente específica da diabete a segunda doença mais prevalente nas crianças brasileiras (VARGAS DM, et al., 2016). Ainda, a DM do tipo 2 (DM2), antes vista como uma desordem quase exclusiva da população adulta, também está se tornando prevalente na população com menos idade, sendo comum ser encontrada nos adolescentes de 12 a 14 anos (GABBAY M, et al., 2003).

A adolescência é a fase entre a infância e a idade adulta, na qual o jovem procura a identidade própria e se aproxima de outros sujeitos que vivem a mesma experiência, consequentemente, se afastando daqueles que não mais se identificam, o que muitas vezes se reflete no distanciamento familiar. Contudo, ao longo dessa jornada em que buscam originalidade, autenticidade e pensamentos próprios, o adolescente pode assumir posturas que até então eram incomuns às da sua infância, como de hiperatividade, isolamento ou timidez (CARRETEIRO TCOC, 2020). 
Partindo desse pressuposto e considerando que a adolescência é uma fase de alterações físicas, comportamentais e sociais, a adesão e continuidade ao tratamento de DCNT em jovens muitas vezes pode não ocorrer de forma satisfatória. Os indivíduos mais novos diagnosticados com DM1, por exemplo, necessitam administrar as suas transformações físicas e psicológicas ao mesmo tempo em que sofrem as dificuldades da própria doença. Logo, podem apresentar sentimentos de rebeldia e apatia com sua condição, tornando-se um desafio maior para os profissionais de saúde responsáveis pela conduta terapêutica e melhoria da qualidade de vida desses pacientes (COLLET N, et al., 2018).

Portanto, o acompanhamento desse grupo de jovens é um processo longo e que requer uma maior atenção das Equipes de Saúde, necessitando a construção de um autocuidado individualizado e que contorne os aspectos físicos e psicossociais que podem interferir na adesão ao tratamento (FLORA MC e GAMEIRO $\mathrm{MGH}, 2016)$.

Dessa forma, reconhecendo a complexidade desse cenário e a importância que um manejo eficaz pode promover na saúde de um indivíduo, essa pesquisa possui como objetivo discutir e revisar sobre os fatores intervenientes na autonomia dos adolescentes diabéticos tipo 1 e a repercussão desses na qualidade de vida.

\section{MÉTODOS}

Trata-se de uma Revisão Integrativa da Literatura, pois consiste numa pesquisa que visa obter conhecimento acerca de determinado assunto por meio de uma ampla análise de estudos anteriores (SOUZA MT, et al., 2010). Para isso, utilizou-se a partir da abordagem qualitativa os descritores "Diabetes Mellitus" AND "Quality of life" OR "Adolescents" na base de dados Scientific Electronic Library Online (SciElo) e no portal da Biblioteca Virtual em Saúde (BVS), sendo selecionados apenas os artigos científicos datados entre o ano de 2010 a 2020 e que apresentassem correlação com o tema.

A pesquisa dos artigos foi realizada entre dezembro de 2020 e fevereiro de 2021. Inicialmente, foram encontrados 5942 artigos, porém apenas 23 destes se encontravam dentro dos critérios de elegibilidade. Os critérios de inclusão adotados para esse estudo foram: abordagem sobre adolescentes com DM1 e qualidade de vida em adolescentes diabéticos. Como critérios de exclusão, adotou-se não utilizar artigos com informações incompletas ou que não abordaram o Brasil, estudos fora do período determinado e irrelevantes para a pesquisa (Figura 1).

Figura 1 - Fluxograma descrevendo os critérios de inclusão e exclusão adotados para o estudo

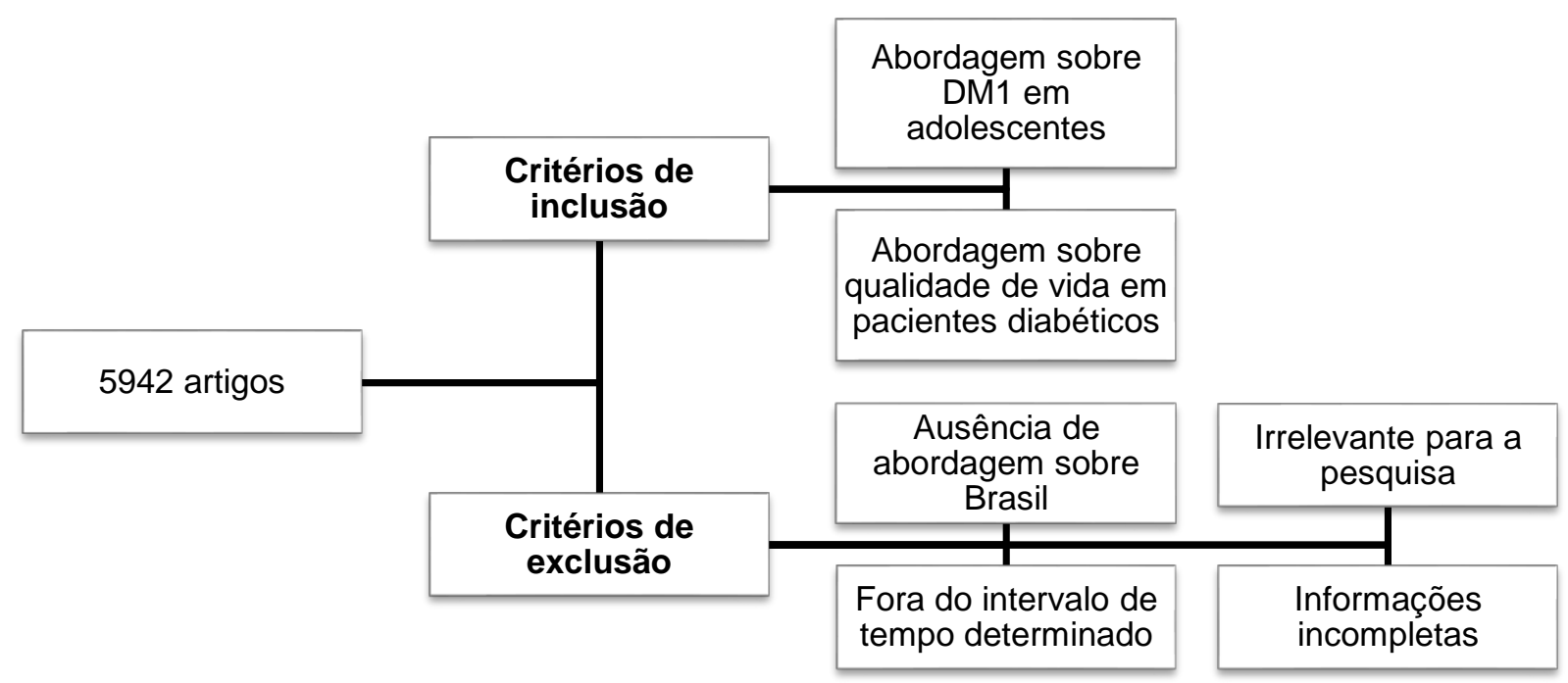

Fonte: Santana IACM, et al., 2021. 


\section{RESULTADOS}

Dentre os 23 artigos selecionados, a maioria diz respeito aos fatores que interferem na qualidade de vida em adolescentes diabéticos. Destes, quatro artigos abordam a importância dos pais no processo do controle glicêmico e desenvolvimento da autonomia no paciente jovem. Além disso, dez artigos apontam para a interferência de fatores emocionais, estruturais e a falta de conhecimento da doença na adesão ao tratamento e qualidade de vida. Há também quatro artigos com conceitos importantes dentro do tema e, por fim, cinco outros abordam os fatores que superam as dificuldades na adesão ao tratamento e, consequentemente, estão associados à melhoria na qualidade de vida (Quadro 1).

Quadro 1 - Síntese e caracterização dos principais achados dos estudos científicos incluídos na revisão.

\begin{tabular}{|c|c|c|}
\hline $\mathbf{N}$ & Autores (Ano) & Principais achados \\
\hline 1 & $\begin{array}{l}\text { ABABLAZA P, et } \\
\text { al. (2017) }\end{array}$ & $\begin{array}{c}\text { Estudo de revisão. Apresenta novas tecnologias que, apesar do alto custo, } \\
\text { estão associadas à redução de complicações pelo melhor controle metabólico } \\
\text { e à melhoria na qualidade de vida. }\end{array}$ \\
\hline 2 & $\begin{array}{l}\text { COLLET N, et al. } \\
\qquad(2018)\end{array}$ & $\begin{array}{l}\text { Pesquisa qualitativa. Expõe a necessidade do pré-adolescente de aceitar e } \\
\text { saber lidar com a nova rotina relacionada à doença, além de ter conhecimento } \\
\text { sobre a doença e seus riscos e receber apoio da rede de cuidado. }\end{array}$ \\
\hline 3 & $\begin{array}{l}\text { COSTA LMFC e } \\
\text { VIEIRA SE (2015) }\end{array}$ & $\begin{array}{l}\text { Estudo de corte transversal. Associa a pior qualidade de vida com o } \\
\text { atendimento na saúde pública, maior tempo de diagnóstico e sedentarismo. }\end{array}$ \\
\hline 4 & $\begin{array}{l}\text { CRUZ DSM, et al } \\
\qquad(2018)\end{array}$ & $\begin{array}{l}\text { Estudo exploratório-descritivo e qualitativo. Esclarece que o acolhimento dos } \\
\text { pais e a boa relação entre família, amigos e equipe favorecem a aceitação da } \\
\text { doença. }\end{array}$ \\
\hline 5 & $\begin{array}{l}\text { DELLA MANNA T, } \\
\text { et al. (2020) }\end{array}$ & $\begin{array}{l}\text { Estudo de revisão. Evidencia que, considerando o aumento na incidência e } \\
\text { prevalência do DM na infância, é necessária constante atualização sobre a } \\
\text { doença, a fim de obter uma educação profissional contínua, em vista de } \\
\text { garantir uma boa qualidade de vida a população jovem. }\end{array}$ \\
\hline 6 & $\begin{array}{l}\text { FLORA MC e } \\
\text { GAMEIRO MGH } \\
(2016)\end{array}$ & $\begin{array}{l}\text { Estudo de corte transversal, descritivo e analítico. Relata haver maior } \\
\text { dificuldade em compreender a importância da hemoglobina glicada e em } \\
\text { ajustar a insulina de acordo com a monitorização por glicemia capilar. }\end{array}$ \\
\hline 7 & $\begin{array}{l}\text { GARCIA LF, et al. } \\
\qquad(2018)\end{array}$ & $\begin{array}{c}\text { Tradução-tradução reversa com análise descritiva. Esclarece que o } \\
\text { instrumento PedsQLTM3.0 é aplicável a crianças / adolescentes brasileiros } \\
\text { com DM1 e seus cuidadores, sendo confiável em indivíduos abaixo de } 11 \\
\text { anos. }\end{array}$ \\
\hline 8 & $\begin{array}{l}\text { MENEZES M, et al. } \\
\qquad(2019)\end{array}$ & $\begin{array}{c}\text { Estudo descritivo, exploratório e quantitativo. Apresenta boa percepção de } \\
\text { qualidade de vida quanto a bullying, autonomia e família devido à boa adesão } \\
\text { e segurança, e baixa qualidade de vida quanto à atividade física, relacionada } \\
\text { à insatisfação física e baixa energia. }\end{array}$ \\
\hline 9 & $\begin{array}{l}\text { PAULA JS, et al. } \\
\qquad(2017)\end{array}$ & $\begin{array}{l}\text { Estudo prospectivo. Evidencia que os níveis médios de glicose têm melhor } \\
\text { relação com a percepção, à curto prazo, da qualidade de vida. }\end{array}$ \\
\hline 10 & $\begin{array}{l}\text { PEREIRA EV, et } \\
\text { al. (2020) }\end{array}$ & $\begin{array}{l}\text { Revisão sistemática. Avalia a relação qualidade de vida com as intervenções, } \\
\text { demonstrando melhora da qualidade de vida nos pacientes do grupo } \\
\text { intervenção em } 40,6 \% \text { dos estudos. }\end{array}$ \\
\hline 11 & $\begin{array}{l}\text { SILVA GA, et al. } \\
\qquad(2020)\end{array}$ & $\begin{array}{l}\text { Estudo de corte transversal, descritivo e analítico. Relata que há um grande } \\
\text { número de solicitações desnecessárias de TOTG, sendo importante a } \\
\text { construção de novos critérios para requerer o procedimento. }\end{array}$ \\
\hline 12 & $\begin{array}{l}\text { SOUZA MA, et al. } \\
\qquad(2019)\end{array}$ & $\begin{array}{c}\text { Estudo de corte transversal. Evidencia que complicações da doença, número } \\
\text { de hospitalizações, de injeções diárias de insulina e classe econômica mais } \\
\text { baixa estão associados à piora na qualidade de vida. }\end{array}$ \\
\hline 13 & $\begin{array}{l}\text { VAZ EC, et al. } \\
\qquad(2018)\end{array}$ & $\begin{array}{l}\text { Revisão sistemática. Evidencia que a contagem de carboidratos tem } \\
\text { benefícios, apesar de limitados, na redução da } \mathrm{HbA1c.}\end{array}$ \\
\hline
\end{tabular}

Fonte: Santana IACM, et al., 2021. 


\section{DISCUSSÃO}

O DM1 é o distúrbio endócrino crônico mais comum na infância, relacionado à alta morbimortalidade e redução da expectativa de vida, principalmente quando há descontinuidade do tratamento (FLORA MC e GAMEIRO MGH, 2016). Caracterizado pela destruição gradual e progressiva de células beta pancreáticas por um processo autoimune, essa doença gera redução na produção de insulina, com necessidade do uso de insulina exógena (COSTA LMFC e VIEIRA SE, 2015).

A etiologia do DM1 ainda é pouco conhecida, porém sabe-se que a combinação de fatores genéticos e ambientais precipitantes, a exemplo de infecções virais, levam a ocorrência de uma resposta imune que pode gerar redução da secreção insulínica. Inicialmente esse quadro não possui uma repercussão clínica, porém, com a evolução da doença, os sinais e sintomas típicos de poliúria, polidipsia, polifagia e perda ponderal costumam aparecer (DELLA MANNA T, et al., 2016).

Nesse cenário, a mudança do quadro clínico de assintomático para sintomático é o principal momento no qual o paciente sente a necessidade de acompanhamento médico, sendo o período mais comum em que 0 diagnóstico é feito. Para isso, além de considerar as manifestações orgânicas, alguns exames laboratoriais são usualmente prescritos para auxiliar na confirmação da doença. Assim, valores elevados da glicemia em jejum (>126mg/dL); do teste de tolerância à glicose oral (>200mg/dL) e da glicemia ao acaso (>200mg/dL) devem servir de alerta para o profissional de saúde (SILVA GA, et al., 2020).

O manejo do DM1 é complexo devido ao seu caráter crônico e rigoroso para atingir o equilíbrio no controle glicêmico de forma assídua, necessitando de condutas diárias de insulinoterapia por múltiplas injeções ou por infusão subcutânea contínua (conhecida como bomba de insulina), dieta com restrição de carboidratos, automonitoramento glicêmico, exercício físico regular e consultas frequentes para monitorização e adequação do tratamento (GARCIA LF, et al., 2018).

Segundo Vaz EC, et al. (2018), o controle do diabetes é avaliado à longo prazo pelos níveis de hemoglobina glicada (HbA1c) que revela o histórico dos níveis glicêmicos do paciente nos últimos dois a três meses. Para mais, pode-se utilizar de medições de glicemia em jejum e após as refeições para avaliação diária, sendo os valores ideais, segundo a Sociedade Brasileira de Diabetes, de $\mathrm{HbA} 1 \mathrm{c}<7,5 \%$; glicemia em jejum 90-145 mg/dL; e glicemia pós-prandial 90-180 mg/dL.

Nessa perspectiva, temos que o manejo adequado desde o diagnóstico permite o controle metabólico e, consequentemente, melhor percepção na qualidade de vida pessoal, social, escolar e profissional dos adolescentes diabéticos e suas famílias. Não obstante, o autocuidado e controle da doença reduzem a perda de produtividade e os custos em saúde, principalmente pela redução de complicações, associando-se, também, ao desenvolvimento econômico (FLORA MC e GAMEIRO MGH, 2016).

Enquanto na infância a responsabilidade pelo tratamento é dos pais, na adolescência o objetivo deve ser de estimular a autonomia do paciente para o controle glicêmico, a fim de que ele encontre maior facilidade no autocuidado ao chegar na vida adulta (COLLET N, et al., 2018; COSTA LMFC e VIEIRA SE, 2015). Com isso, o adolescente deve se adaptar continuamente à doença e ao controle metabólico, que, por ser diário e rigoroso, pode ser um processo exaustivo, gerando dificuldade de aceitação da doença ou desvios no autocuidado, o que gera grande impacto na qualidade de vida (FLORA MC e GAMEIRO MGH, 2016; GARCIA LF, et al., 2018).

Considerando que a transição para a adolescência envolve diversas descobertas e alterações comportamentais, sociais, psicológicas e sentimentais, alguns fatores como dependência dos pais, imaturidade e sentimentos como rebeldia, timidez e apatia podem intervir negativamente no autocuidado do diabetes, levando à descontinuidade no tratamento e ao aumento de complicações da doença (COLLET N, et al., 2018; COSTA LMFC e VIEIRA SE, 2015).

As complicações agudas, relacionadas a episódios hipo ou hiperglicêmicos, assim como o medo de complicações crônicas demonstram interferir na qualidade de vida dos adolescentes diabéticos (PAULA JS, et al., 2016). Por outro lado, a insulinoterapia intensiva por injeções diárias, que evita tais complicações, está, também, associada à pior qualidade de vida dos pacientes, devido ao medo da dor tanto na medição da glicemia capilar, quanto durante a administração de insulina (PEREIRA EV, et al., 2020). 
As complicações crônicas são causadas devido à oscilação glicêmica no sangue, gerando lesões microvasculares, como retinopatia e nefropatia. Por isso, o manejo inadequado da doença aumenta o risco desses agravos, aumentando, também, o risco de mortalidade precoce pela doença (FLORA MC e GAMEIRO $\mathrm{MGH}, 2016)$. Os estudos apontam que o controle glicêmico confere ao paciente maior estabilidade em sua saúde, evitando o desenvolvimento de nefropatias e retinopatias (MENEZES M, et al., 2019).

Estudos se mostram contraditórios quanto à qualidade de vida e o tempo desde o diagnóstico. Apesar de alguns estudos apontarem correlação indiretamente proporcional entre esses fatores por conta da influência do curso da doença nas atividades sociais diárias, outros estudos apontam que a consciência da cronicidade pode auxiliar na adaptação às medidas cotidianas de tratamento e, consequentemente, aumentar a percepção de qualidade de vida (COSTA LMFC e VIEIRA SE, 2015).

Haja vista que o sofrimento causado pela doença crônica é vivido pelo paciente e familiares, os pais podem influenciar na qualidade de vida, tanto de forma negativa, pelo envolvimento excessivo e superproteção quanto ao controle glicêmico, aspecto que se choca com a busca pela independência, natural da adolescência; quanto de forma positiva, auxiliando no desenvolvimento da autonomia necessária no tratamento (MENEZES M, et al., 2019; SOUZA MA, et al., 2019).

Além disso, o fator cronicidade está relacionado à pior qualidade de vida nas famílias em estado de baixa escolaridade e baixo poder socioeconômico, devido à falta de informações quanto à doença, além da incerteza econômica futura, haja vista que o DM1 gera custos diretos e indiretos pela implementação e manutenção do tratamento, principalmente os mais modernos; bem como com o tratamento das complicações agudas ou crônicas (COSTA LMFC e VIEIRA SE, 2015; PEREIRA EV, et al., 2020).

Tendo em vista os fatores citados acima, percebe-se a necessidade e importância da equipe profissional e da educação em saúde no acompanhamento individualizado de adolescentes com DM1 para o controle efetivo da doença e minimização das suas complicações, o que permite a implementação de novos hábitos e adaptação à rotina, bem como a melhoria da qualidade de vida (FLORA MC e GAMEIRO MGH, 2016).

O autocuidado diz respeito à manutenção do estilo de vida saudável por qualquer indivíduo. No entanto, em adolescentes diabéticos, esse conceito adquire grande importância para um tratamento efetivo, sendo diretamente relacionado à autonomia e responsabilidade, pois a autonomia (tomada de iniciativa) permite que - adolescente assuma a responsabilidade pela sua própria saúde, alcançando o autocuidado e, consequentemente, a melhoria na qualidade de vida (FLORA MC e GAMEIRO MGH, 2016; COLLET N, et al., 2018).

O autocuidado apoiado, de acordo com Collet N, et al. (2018), propicia abertura para o protagonismo do usuário em relação à sua própria saúde, e apresenta cinco etapas, conhecidas como "Cinco A's": 1) avaliação de comportamentos, vida social e histórico de saúde, a fim de conhecer o contexto do paciente; 2) aconselhamento, em que serão passadas informações visando a educação em saúde; 3) acordo, que consiste na construção conjunta do plano individualizado de autocuidado; 4) assistência, caracterizada pela identificação de barreiras, auxílio com incentivo com propósito de aperfeiçoar o automonitoramento e rever as metas; e 5) acompanhamento, em que haverá elaboração e execução do monitoramento com o profissional de saúde, a fim de adequar o manejo às possibilidades reais do paciente.

$O$ processo de autonomia poderá ser facilitado se o contexto social e familiar dos adolescentes lhe disponibilizar atenção, buscando conhecer a doença, demostrando interesse pelas ações positivas e motivando a sua iniciativa no tratamento (FLORA MC e GAMEIRO MGH, 2016). Além disso, "quando o adolescente se sente bem consigo mesmo e com os outros, mantém elevada sua autoestima e procura se cuidar. Essa é uma forma de melhorar seu nível glicêmico" (CRUZ DSM, et al., 2018).

Além disso, há ferramentas e tecnologias em saúde que permitem maior autonomia no controle metabólico do paciente diabético, o que proporciona maior conforto e, consequentemente, melhora a percepção da qualidade de vida. São algumas delas: a contagem de carboidratos, a bomba de infusão contínua de insulina subcutânea ou bomba de insulina, o monitoramento contínuo de glicose e o pâncreas artificial (VAZ EC, et al., 2018; PEREIRA EV, et al., 2020; APABLAZA P, et al., 2017). 
A contagem de carboidratos é uma ferramenta simples no tratamento do DM1 que permite maior flexibilidade na escolha alimentar, pois consiste na medição, em gramas, da quantidade de carboidratos por refeição ou no cálculo equivalente de carboidratos por porção de alimento. Tem utilidade, quando associado à medição dos níveis glicêmicos pré-prandiais, na noção da dose de insulina rápida ou regular antes das refeições e, a liberdade de escolha do número de refeições pode melhorar a aceitação da doença (VAZ EC, et al., 2018).

Considerando que o controle glicêmico influencia não apenas na redução de incidência de complicações, mas também na melhoria da qualidade de vida, estudos evidenciam a relação entre os níveis médios de glicose (medido por testes diários de glicemia capilar) para monitorar os tratamentos à curto prazo e melhorar a percepção do paciente quanto à sua autonomia no manejo de insulina (PAULA JS, et al., 2016). O monitoramento contínuo de glicose facilita essa medição por fazê-la de forma contínua e automática. Apesar de não ser capaz de detectar alterações rápidas na glicemia (por captar a glicemia intersticial), essa tecnologia permite o bom controle metabólico e redução de episódios hipoglicêmicos (APABLAZA P, et al., 2017).

O uso de dispositivos para infusão contínua de insulina consiste em um dispositivo que fornece insulina de forma contínua ao tecido subcutâneo, substituindo a secreção fisiológica do pâncreas em momentos de jejum (APABLAZA P, et al., 2017). Este método foi estudado como uma alternativa para melhorar a adesão ao tratamento e controle metabólico, por estarem melhor relacionados à qualidade de vida do que a aplicação de múltiplas injeções diárias (PEREIRA EV, et al., 2020).

Apesar de possuir um alto custo, o "Sensor-Augmented Pump Therapy" (SAPT), que é um sistema integrado entre a bomba de insulina e sensor contínuo de glicose, tem o benefício de permitir o controle metabólico de forma mais sensível, pois atua interrompendo automaticamente a infusão de insulina em episódios hipoglicêmicos e retornando logo após resolução ou em até duas horas após a hipoglicemia (APABLAZA P, et al., 2017).

Já o pâncreas artificial possui maior integração com o monitoramento glicêmico contínuo, pois regula o fornecimento de insulina basal, via subcutânea, de acordo com a medida da glicemia, permitindo maior flexibilidade nas refeições, sem a necessidade de contagem de carboidratos. Apesar disso, o paciente deve informar à equipe de saúde sobre a ingestão de alimentos para que haja a regulação adequada do dispositivo, além da desvantagem já discutida da medição da glicemia intersticial (APABLAZA P, et al., 2017).

Flora MC e Gameiro MGH (2016) evidenciam que a dor para realização da glicemia capilar e o medo da administração de insulina podem estar associados à origem da dificuldade que os adolescentes sentem na gestão do autocuidado. Nesses casos, as tecnologias de bomba infusora de insulina e a utilização de dispositivos de monitorização de glicemias contínuas podem minimizar o impacto da dor, assim como aumentar o conforto e autonomia no manejo da própria doença.

A intervenção deverá, então, considerar as formas do manejo terapêutico e, também, as variáveis socioculturais, familiares e pessoais, trabalhando a educação permanente do paciente quanto à doença. A manutenção do autocuidado vem do acompanhamento individualizado no processo de adaptação dos adolescentes às oscilações glicêmicas através de insulinoterapia, alimentação, exercício físico e monitorização do estado de saúde (FLORA MC e GAMEIRO MGH, 2016).

\section{CONSIDERAÇÕES FINAIS}

As doenças crônicas representam um grave problema de saúde pública, pois geram complicações de saúde muitas vezes irreversíveis. Diante disso, conhecer a patologia e as formas de cuidado da DM1 representam para a vida do adolescente a possibilidade de intervenções que auxiliem na adesão terapêutica e, consequentemente, na melhoria da sua qualidade de vida. Nessa perspectiva, temos que a construção de novos estudos que visam discutir o processo de autocuidado em jovens diabéticos ainda é uma tarefa necessária dentro do âmbito da saúde, pois pode contribuir para o aperfeiçoamento dos profissionais da área a respeito do acolhimento, acompanhamento e manejo adequado desse grupo de pacientes bem como de seus familiares. 


\section{REFERÊNCIAS}

1. APABLAZA P, et al. De la bomba de insulina y el monitoreo continuo de glucosa al páncreas artificial. Rev. méd. Chile, 2017; 145(5): 630-640

2. BRASIL. Estratégias para o cuidado da pessoa com doença crônica: diabetes mellitus. 2013. Disponível em:https://bvsms.saude.gov.br/bvs/publicacoes/estrategias_cuidado_pessoa_diabetes_mellitus_cab36.pdf. Acessado em: 06 de março de 2020.

3. CARRETEIRO TCOC. Reflexões sobre adolescências e a complexidade das comunidades de afeto no processo socioeducativo. Soc. estado., 2020; 35(1): 83-100

4. COLLET N, et al. Autocuidado apoiado no manejo da Diabetes tipo 1 durante a transição da infância para adolescência. Rev. esc. enferm., 2018; 52: e03376

5. COSTA LMFC, VIEIRA SE. Qualidade de vida de adolescentes com diabetes tipo 1. Clinics, 2015; 70(3): 173-179

6. CRUZ DSM, et al. Qualidade de vida relacionada à saúde de adolescentes com dm1- revisão integrativa. Ciênc. saúde coletiva, 2018; 23(3): 973-989

7. DELLA MANNA T, et al. Diabetes mellitus na infância: uma condição emergente no século XXI. Rev. Assoc. Med. Bras., 2016; 62(6): 594-601

8. DUNCAN BB, et al. Doenças Crônicas Não Transmissíveis no Brasil: prioridade para enfrentamento e investigação. Rev Saúde Pública, 2012; 46: 126-34

9. FLORA MC, GAMEIRO MGH. Dificuldades no autocuidado dos adolescentes com diabetes mellitus tipo 1. Rev. Enf. Ref., 2016; 4(11): 31-40

10. GABBAY M, et al. Diabetes melito do tipo 2 na infância e adolescência: revisão da literatura. Jornal de Pediatria, 2003; 79(3): 201-208

11. GARABELI AA, et al. Percepção da qualidade de vida de pacientes diabéticos tipo 1 tratados com análogos de insulina e em revisão medicamentosa com acompanhamento em serviço público de saúde de Ponta Grossa-PR, Brasil. Braz. J. Pharm. Sci., 2016; 52(4): 669-677.

12. GARCIA LF, et al. Translation and validation of Pediatric Quality of Life InventoryTM3.0 Diabetes Module (PedsQLTM3.0 Diabetes Module) in Brazil-Portuguese language. J. Pediatr., 2018; 94: 680-688

13. MARTINS KAKF, et al. Qualidade de vida relacionada à saúde em uma coorte de jovens com diabetes tipo 1. Rev. Assoc. Med. Bras., 2018; 64(11): 1038-1044.

14. MELO SPSC, et al. Doenças crônicas não transmissíveis e fatores associados em adultos numa área urbana de pobreza do nordeste brasileiro. Ciênc. saúde coletiva, 2019; 24(8): 3159-3168

15. MENDES TAB, et al. Diabetes mellitus: fatores associados à prevalência em idosos, medidas e práticas de controle e uso dos serviços de saúde em São Paulo, Brasil. Cad. Saúde Pública, 2011; 27(6): 1233-1243

16. MENEZES M, et al. Qualidade de Vida e Diabetes Mellitus: Autopercepção de Adolescentes de uma Cidade do Sul do Brasil. Psicologia: Teoria e Pesquisa, 2019; 35: e35430

17. PAULA JS, et al. Correlação entre os parâmetros de automonitoramento da glicemia e a percepção da qualidade de vida relacionada à saúde em pacientes com diabetes mellitus tipo 1. Arco. Endocrinol. Metab., 2017; 61(4): 343-347

18. PEREIRA EV, et al. Avaliação da aplicação do Questionário de Qualidade de Vida em Diabetes em pacientes com diabetes mellitus. Arco. Endocrinol. Metab., 2020; 64(1): 59-65

19. RAMOS LBS, et al. Qualidade de Vida, Depressão e Adesão ao Tratamento de Pessoas com Diabetes Mellitus Tipo 2. Rev. bras. ciênc. Saúde, 2017; 21(3): 261-268

20. SILVA GA, et al. Teste oral de tolerância à glicose: solicitações desnecessárias e condições adequadas a realização do teste. J. Bras. Patol. Med. Lab., 2020; 56: e0932020

21. SOUZA MA, et al. Health-related quality of life of adolescents with type 1 diabetes mellitus. Rev. Latino-Am. Enfermagem, 2019; 27: e3210

22. SOUZA MT, et al. Revisão integrativa: o que é e como fazer. Einstein, 2010; 8(1): 102-6

23. THEME FILHA MM, et al. Prevalência de doenças crônicas não transmissíveis e associação com autoavaliação de saúde: Pesquisa Nacional de Saúde, 2013. Revista Brasileira de Epidemiologia online, 2015; 18(2): 83-96

24. VARGAS DM, et al. Perfil Clínico E Epidemiológico De Crianças E Adolescentes Com Diabetes Mellitus 1 Atendidos Na Atenção Secundária Em Blumenau -Sc. Arquivos Catarinenses de Medicina, 2016; 45(3): 58-70

25. VAZ EC, et al. Efetividade e segurança da contagem de carboidratos no tratamento de pacientes adultos com diabetes mellitus tipo 1: revisão sistemática e metanálise. Arco. Endocrinol. Metab., 2018; 62(3): 337-345 